\title{
Refined Prognostic Role of CD68-Positive Tumor Macrophages in the Context of the Cellular Micromilieu of Classical Hodgkin Lymphoma
}

\author{
Alexandar Tzankov Matthias S. Matter Stephan Dirnhofer \\ Department of Pathology, University of Basel, Basel, Switzerland
}

\section{Key Words}

Hodgkin lymphoma - Macrophages • Prognosis •

Micromilieu · Granzyme B · FOXP3 · PD-1

\begin{abstract}
Objective: Classical Hodgkin lymphoma (HL) consists of neoplastic Hodgkin and Reed-Sternberg cells (HRSC) and a nonneoplastic micromilieu that greatly outnumbers the HRSC. Studies on HRSC-related prognostic biomarkers have been unsuccessful, but the microenvironmental composition is of prognostic importance. Recently, the number of CD68-positive macrophages was correlated with adverse survival in $\mathrm{HL}$, and there was a call to validate these results. Methods: We utilized immunohistochemistry to analyze the prognostic importance of the CD68-positive macrophage number compared to other cellular environmental components in an unselected series of $105 \mathrm{HLs}$ in tissue microarrays. Results: Applying a cutoff score of $>0.82 \%$ tumor macrophages, cases with increased numbers showed worse overall survival (mean 185 months, median 192) compared to cases with lower numbers (mean 285 months, median not reached). Eleven of 62 patients with $\leq 0.82 \%$ tumor macrophages died, compared to 19 of 43 with $>0.82 \%(p<0.001)$. The number of macrophages correlated with a low FOXP3-/high granzyme B-/high PD-1-positive micromilieu and patient age, but did not have independent prognostic significance. A combination background score taking into consideration all nega-
\end{abstract}

tive prognostic microenvironmental components (CD68-, PD-1- and granzyme B-positive cells) was of independent prognostic significance $(p=0.002)$. Conclusion: Increased numbers of CD68-positive tumor macrophages indicate an adverse overall outcome in HL.

Copyright $\odot 2011$ S. Karger AG, Basel

\section{Introduction}

Classical Hodgkin lymphoma (cHL) is a lymphoid neoplasm consisting of mononuclear Hodgkin and multinuclear Reed-Sternberg cells (HRSC) embedded in a nonneoplastic background infiltrate of small lymphocytes, eosinophilic and neutrophilic granulocytes, histiocytes, plasma cells, fibroblasts and collagen fibers [1]. It is the paradigm of a formerly lethal neoplastic disease that became curable with the introduction of modern multimodal therapies in the 1970s. With risk- and response-adapted multimodal therapy, over $85 \%$ of cHL patients achieve a long-lasting and complete remission [2]. Nevertheless, about $3 \%$ of stage I and II and about $7 \%$ of stage III and IV cases present with refractory disease, and another $10-$ $15 \%$ relapse, requiring more intensive treatments [3]. A drawback of the impressive achievements in cHL treatment is the increasing late toxicity, particularly the high incidence of secondary malignancies, such as therapy-related myeloid neoplasms, breast and lung cancer and car-

\section{KARGER}

Fax +4161306 1234 E-Mail karger@karger.ch www.karger.com

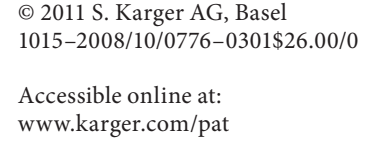

Dr. A. Tzankov

Department of Pathology

University of Basel, Schoenbeinstrasse 40

CH-4031 Basel (Switzerland)

Tel. +41 6132868 80, Fax +416126531 94, E-Mail atzankov@ uhbs.ch 
diovascular diseases [4]. Thus, it is important to be able to predict/identify patients who are treatment resistant and/ or have a higher risk of relapse and find alternative therapeutic options. In addition, patients who might be overtreated by the current treatment regimens must be identified to reduce treatment-associated toxicity.

Prior to the introduction of modern therapy protocols, histological type and nodular sclerosis grading were predictive for disease-specific outcome in cHL [5-8]. Today, disease stage, presence of B symptoms, patient clinical history, including risk factors such as coincidental HIV infection, and laboratory parameters are the most important predictive factors $[9,10]$. Studies searching for a meaningful HRSC-related phenotypic prognostic biomarker in cHL are contradictory and poorly reproducible $[1,11,12]$. Contrary to HRSC-related biomarkers, reactive cellular environment characteristics seem to be of greater prognostic significance in cHL [13-19]. Importantly, cHL lymphomagenesis is more closely related to the cellular background infiltrate than in other lymphomas. HRSC produce many cytokines and chemokines, resulting in the influx and activation of CD4+ cells and histiocytes; conversely, HRSC respond to the chemokines and growth factors produced by these surrounding cells, the latter providing essential feedback signals to stimulate proliferation and inhibit apoptosis in HRSC [20,21]. A possible prognostic role of macrophages and histiocytes in $\mathrm{CHL}$ was suggested decades ago [22-24]. A recent gene expression profiling study on an unselected cHL collective with subsequent immunohistochemical verification on a collective enriched for treatment failures showed an important correlation between the number of CD68-positive macrophages and adverse disease-specific survival (DSS) and worse outcome after secondary high-intensity therapy with autologous stem cell support, but results have thus far not been validated in an independent collective [25]. Therefore, we systematically analyzed the prognostic importance of the number of CD68-positive macrophages in an unselected series of 105 clinically and histopathologically well-characterized cHLs in tissue microarrays (TMAs).

\section{Materials and Methods}

We used a patient set of our previously reported TMA collective $[19,26]$ that met the following criteria: complete clinical documentation, presence of at least 5 unequivocal HRSC/case on the examined slides and presence of at least 1 CD68-positive cell/case on the examined slides. Only 105 of the initial 259 cases could be considered based on these criteria.
Patients

cHL cases $(n=105)$ diagnosed between 1974 and 2000 and reclassified according to the World Health Organization 2008 criteria were collected from the archives of the Institutes of $\mathrm{Pa}$ thology at the University Hospitals of Basel, Switzerland, and Innsbruck, Austria. Paraffin blocks were selected based on availability and preservation. Clinical and follow-up data (summarized in table 1) were obtained from chart reviews. Retrieval of tissue and clinical data was performed according to the regulations of the local institutional review boards and data safety laws. Treatment was either standard or consistent with the investigational protocols active during the time the patients were diagnosed. Patients were staged surgically as stage I or II when radiotherapy (RT) was administered as the sole treatment. Otherwise, patients were staged clinically and treated with risk-adapted treatment strategies according to the protocols of the German Hodgkin Study Group valid at the time of diagnosis. In advanced stages, RT was applied for treatment of residual disease or primary bulky disease $[27,28]$. Disease remission was defined as absence of disease for at least 1 month as determined by laboratory and imaging studies as well as physical examination. Disease relapse was defined as disease progression occurring at least 1 month after achieving disease remission. Treatment failure was defined as disease relapse or primary treatment resistance; the latter was not observed in the present collective. Overall survival (OS) was defined as the time from initial diagnosis to death or the last follow-up examination or other patient contact. DSS was defined as the time from initial diagnosis to death from lymphoma or the last follow-up examination or other patient contact. Relapse-free survival (RFS) was defined as the time from initial diagnosis to disease relapse or the last follow-up examination or other patient contact without imaging, clinical or anamnestic evidence of disease recurrence.

\section{TMA Construction}

The TMAs were constructed and validated as described elsewhere $[19,26]$. Two cores from every sample were arrayed. The construction of the TMAs was approved by the institutional review boards of the participating centers at the time of case submission, and cases have been kept anonymous to meet the legal and ethical requirements for their use in the present study.

\section{Immunohistochemical Evaluation}

The slides were immunohistochemically stained for CD68 using an automated immunostainer (Nexes, Ventana, USA), utilizing the PGM1 antibody from Dako (M0876; 1:200; antigen retrieval with citrate buffer, $\mathrm{pH} 6$, in a microwave oven at $100^{\circ} \mathrm{C}$ for $15 \mathrm{~min}$ ). The total number of cells and CD68-stained cells on each array spot was counted at $\times 200$ magnification. The percentage of CD68-positive cells for each spot was subsequently extracted. The mean percentage over both spots, provided that both contained HRSC, was considered for final statistical analysis, except for cases in which the difference between both spots exceeded $1 \%$ or would lead to reclassification of a case as either negative or positive when considering the cutoff score for CD68-positive tumor macrophages. In both of the latter instances, the HRSC-richer array spot was further considered (see Results). Details on FOXP3, granzyme $\mathrm{B}$ and $\mathrm{PD}-1$ staining have been reported previously [18, 19]. To assess the reproducibility of the CD68 data, comparison between the results of two observers (A.T. and M.S.M.) in one 
third of the cases was performed in a blinded fashion and assessed using interclass correlation coefficients.

\section{Statistics}

Statistical analysis, including data description, was performed using the Statistical Package of Social Sciences 15.0 software (Chicago, Ill., USA). The degree of agreement between observers was evaluated by interclass correlation coefficients, using Cronbach's alpha reliability analysis, with alpha $>0.75$ indicating very good reproducibility. Spearman rank correlation coefficients were used to analyze relationships between markers and/or clinical variables. The Mann-Whitney U and Kruskal-Wallis tests were applied, where appropriate, to assess mean differences between groups. The prognostic performance of the variables and determination of optimal cutoff values of continuous variables were established by receiver operating characteristic (ROC) curves plotting sensitivity versus 1 - specificity. The optimal cutoff point was calculated using Youden's index $(\mathrm{Y})$, where $\mathrm{Y}=$ sensitivity + specificity -1 , since this method can be applied when there is no particular requirement for sensitivity and/or specificity [29]. ROC analysis was applied for OS, DSS and RFS. Survival was then analyzed by the Kaplan-Meier method applying the cutoff values calculated by ROC/Y. Survival results from the present study as well as prognostic factors already established in the study collective $[18,19]$, for which the ROC or Kaplan-Meier methods suggested statistical $(p<0.05)$ or borderline significance $(p<0.1)$, were further considered in the multivariate analysis using a Cox regression model.

\section{Results}

\section{Patients}

Within the median follow-up period of 142 months, $72 \%$ of the patients survived, $10 \%$ died from lymphoma and $18 \%$ died from other causes such as second malignancies $(n=11)$, cardiovascular events $(n=6)$, infections $(\mathrm{n}=2)$ and trauma $(\mathrm{n}=1)$, the former 3 being highly probably attributable to lymphoma therapy. Mean OS was 243 months [95\% confidence interval (CI) 218-268], and mean DSS was 297 months (95\% CI 278-318). The average time to first relapse was 56 months (range 7-240). For the prognostic significance of basic clinicopathological variables, see table 1 .

\section{Assessment of CD68-Positive Tumor Macrophages}

Immunohistochemistry is shown in figure 1. Cronbach's alpha for interobserver reproducibility for a given case was 0.829 . Cronbach's alpha for agreement between results assessed on both TMA spots of a given case was 0.802 . Cases in which the difference between both spots/ cores exceeded $1 \%$ or would have led to reclassification of a case as either negative or positive when considering the cutoff score for CD68-positive tumor macrophages $(\mathrm{n}=$
Table 1. Basic patient characteristics

\begin{tabular}{|c|c|c|c|c|}
\hline \multirow[t]{2}{*}{ Variables } & \multirow[t]{2}{*}{ Cases, n (\%) } & \multicolumn{3}{|c|}{$\begin{array}{l}\text { Prognostic } \\
\text { significance for }\end{array}$} \\
\hline & & OS & DSS & RFS \\
\hline \multicolumn{5}{|l|}{ Gender } \\
\hline Male & $54(51)$ & n.s. & n.s. & n.s. \\
\hline Female & $51(49)$ & & & \\
\hline \multicolumn{5}{|l|}{ Age, years } \\
\hline Mean/median/range & $37 / 34 / 13-87$ & $<0.001$ & n.s. & n.s. \\
\hline$<55$ & $87(83)$ & & & \\
\hline$\geq 55$ & $18(17)$ & & & \\
\hline \multicolumn{5}{|l|}{ Disease stage } \\
\hline I & $16(15)$ & 0.057 & n.s. & 0.049 \\
\hline II & $50(49)$ & & & \\
\hline III & $22(21)$ & & & \\
\hline IV & $17(15)$ & & & \\
\hline \multicolumn{5}{|l|}{ B symptoms } \\
\hline No & $62(59)$ & n.s. & n.s. & n.s. \\
\hline Yes & $43(41)$ & & & \\
\hline \multicolumn{5}{|l|}{ cHL subtype } \\
\hline Nodular sclerosis & $60(57)$ & n.s. & n.s. & n.s. \\
\hline Mixed cellularity & $32(30)$ & & & \\
\hline Lymphocyte rich & $3(3)$ & & & \\
\hline Lymphocyte depleted & $2(2)$ & & & \\
\hline Unclassifiable & $8(8)$ & & & \\
\hline \multicolumn{5}{|l|}{ EBV association } \\
\hline No & $85(81)$ & n.s. & n.s. & n.s. \\
\hline Yes & $20(19)$ & & & \\
\hline \multicolumn{5}{|l|}{ Therapy } \\
\hline RT & $27(26)$ & n.s. & n.s. & n.s. \\
\hline COPP & $18(17)$ & & & \\
\hline $\mathrm{COPP}+\mathrm{RT}$ & $30(29)$ & & & \\
\hline ABVD & $14(13)$ & & & \\
\hline $\mathrm{ABVD}+\mathrm{RT}$ & $16(15)$ & & & \\
\hline \multicolumn{5}{|l|}{ Follow-up, months } \\
\hline Mean/median/range & $\begin{array}{r}146 / 142 / \\
12-331\end{array}$ & & n.a. & \\
\hline Relapses & 29 & n.s. & $<0.001$ & n.a. \\
\hline \multicolumn{5}{|l|}{ Causes of death } \\
\hline Death with/from disease & 10 & & n.a. & \\
\hline Second malignancy & 11 & & & \\
\hline Cardiovascular disease & 6 & & & \\
\hline Other (infection, trauma) & 3 & & & \\
\hline
\end{tabular}

$\mathrm{EBV}=$ Epstein-Barr virus; n.a. = not applicable; n.s. = not significant. 
Fig. 1. A cHL with $<0.82 \%$ CD68-positive tumor macrophages. B A case with $>0.82 \%$ CD68-positive tumor macrophages. Immunoperoxidase stain. $\times 200$.

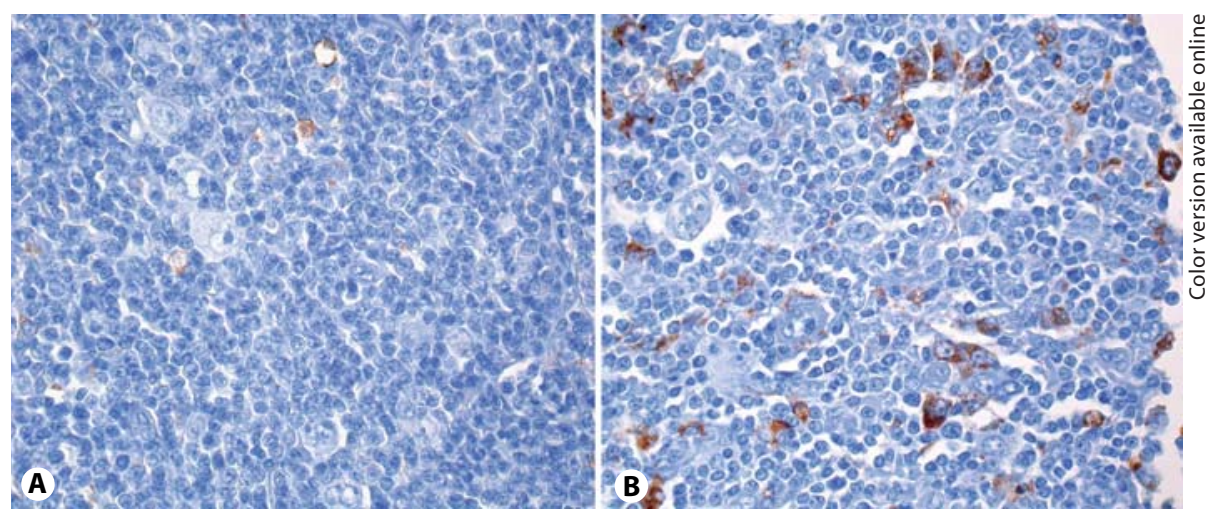

26) were reanalyzed on a double-headed microscope by both investigators. The different distribution of CD68positive tumor macrophages could be reproduced after reevaluation in all these cases and was attributable to the absence or paucity of HRSC on either the first or second arrayed spot. Importantly, in these cases, specific consideration of the prognostic value of the number of CD68positive tumor macrophages assessed on either the first or the second arrayed spot showed that only the number assessed on the spot containing HRSC or enriched in HRSC was of prognostic importance. In such equivocal cases, results assessed on the HRSC-richer array spot were considered further. For descriptive results, see table 2 .

\section{Correlations}

CD68-positive tumor macrophage numbers correlated with patient age $(\rho=0.484, p<0.001 ; 16$ of 18 patients aged $\geq 55$ years had increased numbers of CD68-positive tumor macrophages compared to 28 of 87 patients younger than 55) and with the number of tumor-infiltrating granzyme B-positive lymphocytes $(\rho=0.260, p=0.025)$ and correlated inversely with the number of tumor-infiltrating FOXP3-positive lymphocytes $(\rho=-0.351, \mathrm{p}=$ $0.004)$. There were no other significant correlations.

\section{Prognostic Factors}

ROC analysis suggested a prognostic role for OS, but not DSS or RFS, for the numbers of CD68-positive tumor macrophages $(\mathrm{p}=0.038)$ and granzyme $\mathrm{B}-(\mathrm{p}=0.012)$, PD-1- $(\mathrm{p}=0.027)$ and FOXP3-positive tumor-infiltrating lymphocytes $(p=0.036)$. A prognostic role for RFS and DSS was also found for FOXP3-positive tumor-infiltrating lymphocytes ( $\mathrm{p}=0.012$ and 0.059 , respectively) $[18,19]$. The respective ROC curves considering OS are
Table 2. Quantitative results

\begin{tabular}{lrlll}
\hline cHL subtype & $\begin{array}{l}\text { Cases } \\
\mathrm{n}\end{array}$ & \multicolumn{2}{l}{$\begin{array}{l}\text { CD68+ tumor } \\
\text { macrophages, } \%\end{array}$} & \multirow{2}{*}{$\begin{array}{c}\text { Cases with } \\
\text { mo.82\% }\end{array}$} \\
\cline { 3 - 4 } & & mean \pm SD & range & \\
\hline Nodular sclerosis & 60 & $1.5 \pm 2.2$ & $0.0-7.9$ & $21(35 \%)$ \\
Mixed cellularity & 32 & $2.0 \pm 2.3$ & $0.0-8.6$ & $18(60 \%)$ \\
Lymphocyte rich & 3 & $0.1 \pm 0.07$ & $0.0-0.13$ & 0 \\
Lymphocyte depleted & 2 & $1.3 \pm 0.9$ & $0.7-2.0$ & $1(50 \%)$ \\
Unclassifiable & 8 & $1.4 \pm 1.6$ & $0.0-3.3$ & $3(38 \%)$ \\
p value & & n.s. & & n.s. \\
\hline
\end{tabular}

n.s. = Not significant.

shown in figure 2. With respect to OS, the area under the ROC curve for CD68-positive macrophages was 0.660 (95\% CI 0.514-0.806), indicating that consideration of CD68-positive macrophage numbers is a significant improvement over a random guess. The $\mathrm{Y}$ value pointed to $>0.82 \%$ CD68-positive tumor macrophages as being the most relevant cutoff score with respect to OS, with a prognostic sensitivity of $74 \%$ and a specificity of $65 \%$. Applying this cutoff score, Kaplan-Meier analysis showed worse OS for cHL with increased numbers of CD68-positive tumor macrophages (mean 185 months, 95\% CI 142-227, median 192 months) compared to cases with lower numbers (mean 285 months, 95\% CI 259-311, median not reached). Eleven of 62 patients with $\leq 0.82 \%$ CD68-positive tumor macrophages died, compared to 19 of 43 with $>0.82 \%$ CD68-positive tumor macrophages $(\mathrm{p}<0.001$; fig. 3$)$. There were significant differences with regard to the causes of death between cases with in- 


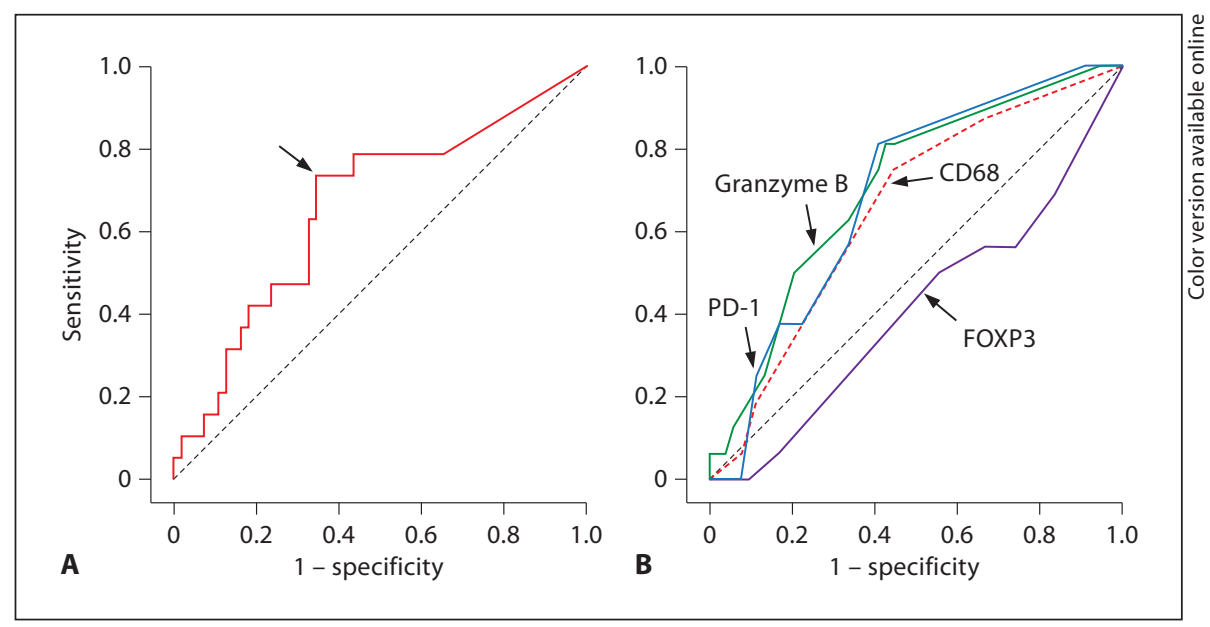

Fig. 2. A ROC curve of the prognostic performance of CD68-positive macrophage numbers in CHL. The area under the ROC of 0.660 indicates that consideration of the CD68-positive macrophage number is $16 \%$ more accurate in predicting survival in cHL than a random guess (dotted line). The curve point (arrow) at a sensitivity of $0.74(74 \%)$ and specificity of 0.65 (65\%; 1 - specificity $=0.35)$ is at the greatest distance from the reference line. B ROC curves of the prognostic performance of CD68-positive macrophage numbers compared to relative amounts of FOXP3-, granzyme $\mathrm{B}$ - and $\mathrm{PD}-1$-positive tumor-infiltrating lymphocytes in cHL. The curve shape of CD68 differs from that in $\mathbf{A}$ due to the consideration of these additional factors. Note the decreasing area under the ROC from granzyme B to CD68 and the reciprocal (inverse) deviation of the FOXP3 curve, reflecting its positive prognostic significance. The larger area under the ROC points to granzyme B-positive cell numbers as the most promising prognostic factor; while PD-1 has significant shortcomings with regard to specificity (note negative/inverse deviations up to coordinates 0.1 ; 0.0 ) but is the most sensitive marker (note that from coordinates $0.4 ; 0.8$ its ROC curve overbears all other curves), CD68 number determination is not better than a random guess (unlabeled, dotted line) up to coordinates $0.1 ; 0.1$. creased and decreased tumor macrophages, with a particular accumulation of second malignancies and infections among those patients with increased tumor macrophages (table 3). Rounding up of the cutoff score from $\leq 0.82$ to $\leq 1 \%$, which we assumed to represent a more practicable cutoff score, would have reclassified 3 of the studied cases from the prognostically adverse to the prognostically favorable group. Since 2 of these patients died from their disease, ROC analysis indicated that application of such a cutoff score rather than $\leq 0.82 \%$ to our collective would lead to a drop in prognostic sensitivity to $63 \%$ without increasing the specificity. Thus, though we acknowledge that a cut-off score of $1 \%$ is more practical, for the purposes of our study, we strictly adhered to the $\leq 0.82 \%$ cutoff, as suggested by ROC/Y. Application of the cutoffs suggested by Steidl et al. [25], i.e. 0 to $<5 \%, 5-25 \%$ and $>25 \%$, led to analogous results, but the absolute number of the collective with a CD68-positive cell number over the cutoff was smaller; thus, the prognostic specificity of adverse events for a cutoff of $\geq 5 \%$ CD68-positive tumor macrophages rose to $92 \%$, but the sensitivity dropped to $16 \%$. In our collective, which was not specifically enriched for treatment fail-
Table 3. Distribution of causes of death with respect to numbers of CD68-positive tumor macrophages

\begin{tabular}{llllll}
\hline $\begin{array}{l}\text { CD68+ tumor } \\
\text { macrophages }\end{array}$ & \multicolumn{4}{l}{ Cause of death } \\
\cline { 2 - 6 } & $\begin{array}{l}\text { lym- } \\
\text { phoma }\end{array}$ & $\begin{array}{l}\text { second ma- } \\
\text { lignancy }\end{array}$ & $\begin{array}{l}\text { cardiovascu- } \\
\text { lar disease }\end{array}$ & $\begin{array}{l}\text { infec- } \\
\text { tion }\end{array}$ & $\begin{array}{l}\text { trau- } \\
\text { ma }\end{array}$ \\
\hline $\begin{array}{l}\text { Increased } \\
\begin{array}{l}\text { Decreased } \\
\text { p value }\end{array}\end{array}$ & 5 & 8 & 3 & 2 & 1 \\
\hline
\end{tabular}

ures, we did not observe a single case with $>9 \%$ CD68positive tumor macrophages.

When analyzed in subgroups according to the clinicopathological variables listed in table 1 , the negative prognostic effect of an increased number of CD68-positive tumor macrophages retained its significance for both genders (males $p=0.008$, females $p=0.015$ ), patients younger than 55 years $(p=0.046)$, stage II $(p=0.001)$ and III disease $(p=0.09)$, patients with and without B symptoms ( $\mathrm{p}=0.016$ and 0.013 , respectively), $\mathrm{cHL}$ of the mixed 


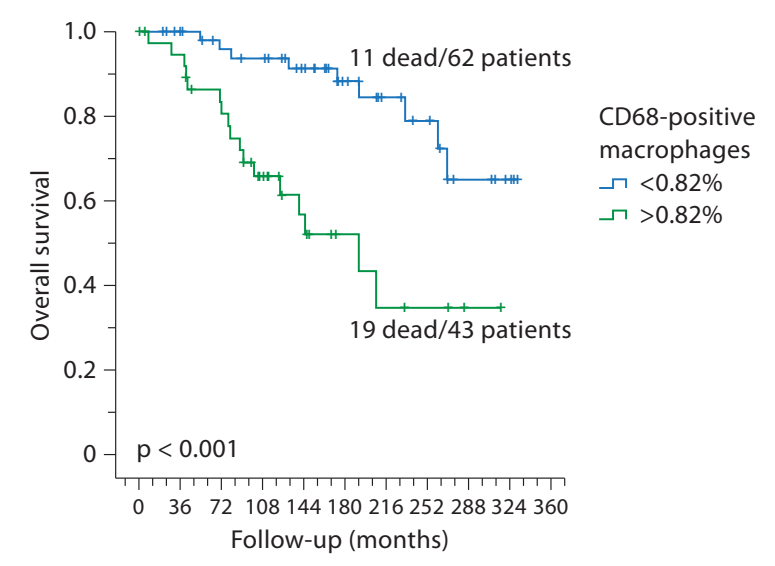

Fig. 3. Kaplan-Meier OS curves of cHLs with respect to the number of CD68-positive tumor macrophages. The p value was calculated by applying the log-rank test.

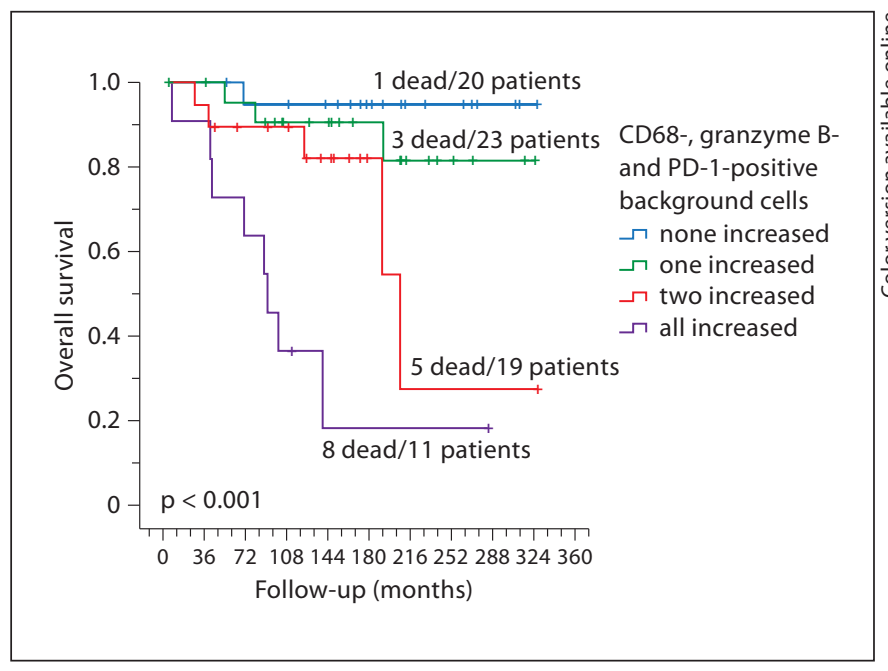

Fig. 4. Kaplan-Meier OS curves of cHLs with respect to cellular background composition. The p value was calculated by applying the log-rank test. The total number of patients $(n=73)$ is lower than that in figure $3(n=105)$ due to uninformative TMA spots for granzyme B- and/or PD-1-positive cellular contents in 32 cases.

cellularity subtype ( $\mathrm{p}=0.008)$, cHL without Epstein-Barr virus association $(\mathrm{p}<0.001)$ and patients treated only with chemotherapy ( $p=0.002)$, especially those treated with cyclophosphamide, vincristine, procarbazine and prednisone (COPP; $\mathrm{p}=0.001)$, and was less pronounced, in patients treated with adriamycin, bleomycin, vinblastine and dacarbazine (ABVD; $\mathrm{p}=0.079$ ) and with COPP plus RT ( $p=0.019)$. However, the shape of the respective Kaplan-Meier curves also suggested a negative prognostic effect of an increased number of CD68-positive tumor macrophages in the subgroups in which statistical significance was not reached. Importantly, the prognostic effects of CD68-, PD-1- and granzyme B-positive cellular contents were cumulative (fig. 4).

In a multivariable analysis considering patient age, disease stage and numbers of FOXP3-, granzyme B- and PD-1-postive tumor-infiltrating lymphocytes, only age, in particular $\geq 55$ years, appeared to be of independent prognostic significance for OS $(p=0.046)$. A combination background score considering all prognostically negative microenvironmental components, i.e. CD68-, PD-1- and granzyme B-positive cell numbers, was of independent prognostic significance $(\mathrm{p}=0.002$; relative risk $2.63,95 \%$ CI 1.41-4.93) compared to age ( $\mathrm{p}=$ not significant; relative risk 2.07) and disease stage ( $\mathrm{p}=$ not significant; relative risk 1.31). Adding CD68 to PD1 and granzyme B made it possible to identify a subgroup of cHLs with an excellent prognosis if all cellular micromilieu components were decreased (1 death with/from lymphoma out of 20 patients) as well as a group with a dismal prognosis if all components were increased (2 deaths with/from lymphoma and 6 deaths from a second malignancy out of 11 patients).

\section{Discussion}

Steidl et al. [25] recently reported that increased CD68positive tumor macrophage numbers have a major impact on the prognosis of cHL. Here, we present the first study to address the reproducibility of their findings. We were able to principally confirm the data of Steidl et al. [25] as well as the findings of Ree and Kadin [23], reported 25 years ago, in our collective, which was not specifically enriched for treatment failures. This lack of enrichment for treatment failures as well as the application of the more specific PGM1 anti-CD68 antibody instead of the KP1 antibody, as applied by Steidl et al. [25], might be the primary reasons for the slightly lower CD68-positive tumor macrophage numbers observed in our collective. In addition, we refined the role of this single biomarker in the context of the cellular micromilieu of cHL. We observed an adverse prognostic significance of an increased number of CD68-positive tumor macrophages with respect to OS. This effect was particularly pronounced in 
mixed cellularity cases, in patients younger than 55 years, in stage II and III disease and in cases without EpsteinBarr virus association. With regard to treatment, especially patients treated by COPP-based therapy, either with or without RT, as well as those treated by chemotherapy alone (either COPP or ABVD), without RT, there was a negative prognostic effect of CD68-positive cell numbers. The advantages of our study are as follows: (1) no preselection of the cases, in particular no enrichment for treatment failures; (2) very long follow-up period (median 12 years); (3) ROC curve-based determination of prognostic cutoff values with both maximum specificity and sensitivity without the need for arbitrary cutoff scores; (4) extensive previous phenotypic cellular background and HRSC characterization of the studied collective $[18,19,26,30]$, and (5) distribution of cHL subtypes reflecting that of the general population.

On the other hand, the limitations of our study include the following: (1) this was a retrospective study with a long period of sample collection (1974-2000) that could include possible bias due to different treatments applied within this period and (2) lack of data on International Prognostic Score and secondary treatment. Importantly, the first and the last point among the advantages as well as the first point among the disadvantages might explain why a prognostic effect with regard to DSS and RFS was not observable in our collective compared to that of Steidl et al. [25], the latter being enriched for treatment failures with a particularly low number of mixed cellularity cases.

Beyond the general reproduction of the results of Steidl et al. [25], the new findings of our study are: (1) discovery in a general cHL collective of a cutoff score of $0.82 \%$ (for practical purposes 1\%) CD68-positive tumor macrophages for OS prognostication, suggesting that at diagnostic determination the histopathologist should recognize whether on average every 100th cell from the cellular background represents a CD68-positive macrophage; (2) CD68-positive cellular contents are prognostically relevant only if assessed in HRSC-containing or -enriched areas (hot spots) rather than in whole tissue specimens; (3) CD68-positive tumor macrophage numbers correlate with a cellular background (low FOXP3/high granzyme $\mathrm{B}$ and PD-1 cellular background) that is usually associated with an unfavorable outcome in cHL $[16-19,30]$, and (4) a particular association of increased CD68-positive tumor macrophage numbers at diagnosis with deaths from second malignancies and infections. Other significant correlations include that between CD68-positive tumor macrophage numbers and patient age in $\mathrm{CHL}$, as well as overrepresentation of cHLs with mixed cellularity and lymphocyte depletion in cases with increased CD68-positive macrophages, which is in agreement with observations from more than 2 decades ago [23].

The reason for the observed prognostic significance of CD68-positive tumor macrophage contents with regard to cHL outcome are unknown, but it probably reflects a particular inflammatory tumor microenvironment or individual immunologic or immunogenetic imprinting [31]. Direct effects of the respective cellular components can also be assumed. For example, it has been suggested that tumors may use the power of granzyme B-positive lymphocytes to their advantage, because mice lacking granzyme B clear tumor grafts more efficiently than do wild-type mice [32]. At least in our collective, CD68-positive tumor macrophage numbers were not an independent prognostic factor in $\mathrm{CHL}$ compared to age, disease stage and numbers of FOXP3-, granzyme B- and PD-1positive tumor-infiltrating lymphocytes. When considered together with other microenvironmental components, namely granzyme-B- and PD-1-positive cells, CD68-positive cells considerably increased the specificity and sensitivity of prognosis in cHL independent of patient age or disease stage being a particular indicator of an increased risk for patients to die of a second malignancy or infection. This supports our assumption that CD68-positive tumor macrophages represent an important, but not exclusive, 'player' in the unfavorable microenvironmental signature probably composed of a limited number of other cytotoxic and regulatory immune cells, the puzzling roles of which remain to be elucidated.

To summarize, increased numbers of CD68-positive tumor macrophages indicate an adverse overall outcome in CHL. For the purpose of prognostication, CD68-positive cell numbers should be assessed in HRSC-rich cHL areas. Increased CD68-positive tumor macrophage counts correlate with a cellular background (low FOXP3/ high granzyme B and PD-1 cells) that is usually associated with an unfavorable outcome in cHL. Prospective studies on cHL collectives treated with modern modalities without selection bias are needed to establish the prognostic importance of the different microenvironmental components. 


\section{References}

1 Tzankov A, Dirnhofer S: Pathobiology of classical Hodgkin lymphoma. Pathobiology 2006;73:107-125.

$\checkmark 2$ Diehl V, Engert A, Re D: New strategies for the treatment of advanced-stage Hodgkin's lymphoma. Hematol Oncol Clin North Am 2007;21:897-914.

$\checkmark 3$ David KA, Mauro L, Evens AM: Relapsed and refractory Hodgkin lymphoma: transplantation strategies and novel therapeutic options. Curr Treat Options Oncol 2007;8: 352-374.

$\checkmark 4$ Landgren O, Caporaso NE: New aspects in descriptive, etiologic, and molecular epidemiology of Hodgkin's lymphoma. Hematol Oncol Clin North Am 2007;21:825-840.

$\checkmark 5$ MacLennan KA, Bennett MH, Tu A, Hudson BV, Easterling MJ, Hudson GV, Jelliffe AM: Relationship of histopathologic features to survival and relapse in nodular sclerosing Hodgkin's disease. A study of 1659 patients. Cancer 1989;64:1686-1693.

-6 Lukes RJ, Butler JJ, Hicks EB: Natural history of Hodgkin's disease as related to its pathologic picture. Cancer 1966;19:317-344.

$\checkmark 7$ Keller AR, Kaplan HS, Lukes RJ, Rappaport $\mathrm{H}$ : Correlation of histopathology with other prognostic indicators in Hodgkin's disease. Cancer 1968;22:487-499.

8 Hess JL, Bodis S, Pinkus G, Silver B, Mauch P: Histopathologic grading of nodular sclerosis Hodgkin's disease. Lack of prognostic significance in 254 surgically staged patients. Cancer 1994;74:708-714.

$\checkmark 9$ Hasenclever D, Diehl V: A prognostic score for advanced Hodgkin's disease. International Prognostic Factors Project on Advanced Hodgkin's Disease. N Engl J Med 1998;339:1506-1514.

$>10$ Josting A, Wolf J, Diehl V: Hodgkin disease: prognostic factors and treatment strategies. Curr Opin Oncol 2000;12:403-411.

-11 Tzankov A, Krugmann J, Fend F, Fischhofer M, Greil R, Dirnhofer S: The prognostic value of CD20 expression in classical Hodgkin Lymphoma - a clinico-pathological study of 119 cases. Clin Cancer Res 2003;9:13811386.

-12 Krugmann J, Tzankov A, Gschwendtner A, Fischhofer M, Greil R, Fend F, Dirnhofer S: Longer failure-free survival interval of Epstein-Barr virus-associated classical Hodgkin's lymphoma: a single-institution study. Mod Pathol 2003; 16:566-573.
13 Poppema S, Potters M, Emmens R, Visser L, Van den Berg A: Immune reactions in classical Hodgkin's lymphoma. Semin Hematol 1999;36:253-259.

14 Poppema S, Van den Berg A: Interaction between host $\mathrm{T}$ cells and Reed-Sternberg cells in Hodgkin lymphomas. Semin Cancer Biol 2000; $10: 345-350$

15 Oudejans JJ, Jiwa NM, Kummer JA, Ossenkoppele GJ, van Heerde P, Baars JW, Kluin PM, Kluin-Nelemans JC, van Diest PJ, Middeldorp JM, Meijer CJ: Activated cytotoxic T cells as prognostic marker in Hodgkin's disease. Blood 1997;89:1376-1382.

16 Ten Berge RL, Oudejans JJ, Dukers DF, Meijer JW, Ossenkoppele GJ, Meijer CJ: Percentage of activated cytotoxic T-lymphocytes in anaplastic large cell lymphoma and Hodgkin's disease: an independent biological prognostic marker. Leukemia 2001;15:458464.

17 Alvaro T, Lejeune M, Salvado MT, Bosch R, Garcia JF, Jaen J, Banham AH, Roncador G, Montalban C, Piris MA: Outcome in Hodgkin's lymphoma can be predicted from the presence of accompanying cytotoxic and regulatory T cells. Clin Cancer Res 2005;11: 1467-1473.

18 Muenst S, Hoeller S, Dirnhofer S, Tzankov A Increased PD-1+ tumor-infiltrating lymphocytes in classical Hodgkin lymphoma substantiate reduced overall survival. Hum Pathol 2009;40:1715-1722.

19 Tzankov A, Meier C, Hirschmann P, Went P, Pileri SA, Dirnhofer S: Correlation of high numbers of intratumoral FOXP3+ regulatory $\mathrm{T}$ cells with improved survival in germinal center-like diffuse large B-cell lymphoma, follicular lymphoma and classical Hodgkin's lymphoma. Haematologica 2008; 93:193-200

20 Van den Berg A, Visser L, Poppema S: High expression of the CC chemokine TARC in Reed-Sternberg cells. A possible explanation for the characteristic T-cell infiltrate in Hodgkin's lymphoma. Am J Pathol 1999; 154:1685-1691.

21 Skinnider BF, Mak TW: The role of cytokines in classical Hodgkin lymphoma. Blood 2002;99:4283-4297.
22 Schiffer CA, Levi JA, Wiernik PH: The significance of abnormal circulating cells in patients with Hodgkin's disease. Br J Haematol 1975;31:177-183.

$>23$ Ree HJ, Kadin ME: Macrophage-histiocytes in Hodgkin's disease. The relation of peanutagglutinin-binding macrophage-histiocytes to clinicopathologic presentation and course of disease. Cancer 1985;56:333-338.

24 Coppleson LW, Rappaport H, Strum SB, Rose J: Analysis of the Rye classification of Hodgkin's disease. The prognostic significance of cellular composition. J Natl Cancer Inst 1973;51:379-390.

25 Steidl C, Lee T, Shah SP, et al: Tumor-associated macrophages and survival in classic Hodgkin's lymphoma. N Engl J Med 2010; 362:875-885

26 Tzankov A, Zimpfer A, Went P, Maurer R, Pileri SA, Geley S, Dirnhofer S: Aberrant expression of cell cycle regulators in Hodgkin and Reed-Sternberg cells of classical Hodgkin lymphoma. Mod Pathol 2005;18:90-96.

27 Greil R, Holzner B, Kemmler G, et al: Retrospective assessment of quality of life and treatment outcome in patients with Hodgkin's disease from 1969 to 1994. Eur J Cancer 1999;35:698-706.

28 Fong D, Steurer M, Greil R, et al: Hodgkin lymphoma in Tyrol - a population-based study. Ann Hematol 2009;88:449-456.

29 Tzankov A, Zlobec I, Went P, Robl H, Hoeller S, Dirnhofer S: Prognostic immunophenotypic biomarker studies in diffuse large B cell lymphoma with special emphasis on rational determination of cut-off scores. Leuk Lymphoma 2010;51:199-212.

30 Adams H, Campidelli C, Dirnhofer S, Pileri SA, Tzankov A: Clinical, phenotypic and genetic similarities and disparities between post-transplant and classical Hodgkin lymphomas with respect to therapeutic targets. Expert Opin Ther Targets 2009;13:11371145.

-31 Enblad G, Molin D, Glimelius I, Fischer M, Nilsson G: The potential role of innate immunity in the pathogenesis of Hodgkin's lymphoma. Hematol Oncol Clin North Am 2007;21:805-823

-32 Cao X, Cai SF, Fehniger TA, et al: Granzyme $\mathrm{B}$ and perforin are important for regulatory T cell-mediated suppression of tumor clearance. Immunity 2007;27:635-646. 